\title{
Pretreatment with a GnRH Agonist and Hormone Replacement Treatment Protocol Could not Improve Live Birth Rate for PCOS Women Undergoing Frozen-Thawed Embryo Transfer Cycles: A Retrospective Cohort Study using Propensity Score Matching
}

Xitong Liu

Northwest Women's and Children's Hospital

Juanzi Shi

Northwest Women's and Children's Hospital

Haiyan Bai ( $\sim 295784720 @ q q . c o m$ )

Northwest Women's and Children's hospital

Research article

Keywords: Frozen-thawed embryo transfer, Endometrial preparation, Live birth rate, Polycystic ovary syndrome

Posted Date: July 30th, 2021

DOI: https://doi.org/10.21203/rs.3.rs-757802/v1

License: (c) (i) This work is licensed under a Creative Commons Attribution 4.0 International License. Read Full License 


\section{Abstract}

Background: The optimal means to prepare the endometrial preparation for PCOS patients is a topic of ongoing controversy. Our objective was to compare the clinical outcomes of frozen-thawed embryo transfer (FET) with and without pretreatment gonadotropin-releasing hormone agonist ( $\mathrm{GnRHa}$ ) in polycystic ovary syndrome (PCOS) patients.

Methods: In this retrospective cohort study, we evaluated 1638 consecutive cycles between June 2014 and December 2017 in the Assisted Reproduction Center of Northwest Women's and Children's Hospital. The patients who underwent FET were assigned to two groups as follows: hormone replacement treatment (HRT) group, comprising 1115 cycles; HRT with GnRHa pretreatment (GnRHa + HRT), comprising 523 cycles. Patients using GnRHa + HRT ( $n=519)$ were matched with 519 patients using HRT.

Results: The live birth rate was higher in the GnRHa + HRT group compared with the HRT group with no significant difference $(60.4 \%$ vs $55.5 \%, p=0.062)$. The clinical pregnancy rate $(72.74 \%$ vs $75.33 \%)$, miscarriage rate $(14.26 \%$ vs $13.96 \%)$ and ectopic pregnancy rate $(0.36 \%$ vs $0.38 \%)$ were similar between the two groups. The preterm birth rate in HRT was lower than GnRHa + HRT (14.08\% vs $20.08 \%)$. There were no significant interactions in any of the subgroups ( $p>0.05$ for all comparisons). In the unadjusted model and adjusted model, the live birth rate was comparable between GnRHa + HRT and HRT group (OR $1.22,95 \% \mathrm{Cl}, 0.99-1.51, \mathrm{p}=0.062$; a OR 1.56, 95\% Cl, 1.001-2.41, p=0.048). Similar results were obtained after propensity score matching in the entire cohort.

Conclusions: GnRHa pretreatment could not improve the live birth rate in women with PCOS.

\section{Background}

Polycystic ovary syndrome (PCOS) is a common endocrine syndrome and affects between 8 and $18 \%$ of women [1]. It is characterized by a combination of the following three criteria: oligoovulation or anovulation, polycystic ovaries, and hyperandrogenism. This syndrome results in luteinizing hormone $(\mathrm{LH})$ and follicle-stimulating hormone (FSH) ratio imbalance, cardiovascular diseases, obesity, infertility, and other health issues.

In recent years, the freeze-all policy of cryopreserving all embryos produced in an IVF cycle to transfer later has become more appropriate when there is a risk of ovarian hyperstimulation syndrome (OHSS), high progesterone level, preimplantation genetic testing (PGT), abnormal endometrium, and other conditions. Studies have demonstrated comparable outcomes between fresh and frozen-thawed embryo transfer (FET) [2-4]. As national consensus continues to endorse single embryo transfer [5, 6], more supernumerary embryos are available for cryopreservation. Patients with PCOS have a greater ovarian response to gonadotropin stimulation than normal response women undergoing IVF. Women with PCOS tend to have more oocytes retrieved and an elevated estradiol level. The altered hormone level may adversely affect endometrial receptivity in fresh embryo transfer. FET has been widely used for PCOS patients to minimize the risk of OHSS. Protocols described in FET include natural cycle, hormone 
replacement treatment (HRT) cycle, and down-regulation with GnRH agonists (GnRHa). Thus, HRT with and without GnRHa becomes the most common method of endometrial preparation for PCOS patients. HRT regimens avoid the cost of $\mathrm{GnRHa}$ and may have similar clinical outcomes. However, the $\mathrm{GnRHa}$ cycle offers the most control over the timing of the cycle and minimizes cancellation rates. The GnRHa cycles also eliminate the possible detrimental role of high LH on the oocyte quality and implantation rate.

Currently, the optimal means to prepare the endometrial preparation for PCOS patients is a topic of ongoing controversy. Most studies focused on ovulatory women instead of PCOS patients, and existing studies have been conflicting. Therefore, we aimed to evaluate the live birth rate between two different endometrial preparations (HRT and GnRHa + HRT) for PCOS patients.

\section{Methods}

\section{Study design and patients}

This was a consecutive, retrospective cohort study of 1638 cycles undergoing FET between June 2014 and December 2017 in the Center for Assisted Reproductive Technology of Northwest Women's and Children's Hospital, China. The selection of the study population was shown in Fig. 1. Women who underwent the first cycle of FET were considered eligible for the study if they were diagnosed with PCOS according to Rotterdam criteria and had previous in vitro fertilization cycles with embryo cryopreservation, regardless of age, diagnosis, embryo stage, or the number of transferred embryos. We excluded patients who were not diagnosed with PCOS and multiple FET cycles.

\section{Propensity Score Matching (Psm)}

Given the differences in the baseline characteristics between the two groups, propensity-score matching was used to identify a cohort of women with similar baseline characteristics. A propensity score for endometrial preparation was estimated applying a multivariable logistic regression model, with female age at embryo transfer and BMI as covariates. Matching was performed with the use of a 1:1 matching protocol without replacement, with a caliper width equal to 0.05 of the standard deviation of the logit of the propensity score.

\section{Endometrial Preparation Protocols}

Hormone replacement treatment (HRT) cycle Supplementation with oral estrogens (4-6mg/day; Progynova; Bayer Schering Pharma AG, Berlin, Germany) was administered on day 2-5 of the menstrual cycle. Serum progesterone $(P)$ level and ultrasound were performed 10-12 days later. If endometrial thickness $>7 \mathrm{~mm}$ and $\mathrm{P}<1.5 \mathrm{ng} / \mathrm{ml}$, progesterone was administered, and FET was scheduled. Embryo transfer was performed after 3 days (cleavage stage embryos) or 5 days (blastocyst stage embryos). 
GnRHa + HRT cycle GnRHa (3.75mg; Diphereline, Ipsen Pty Ltd, France) was injected on day 2-5 of menstruation. Oral estrogens were administered 30 days after $\mathrm{GnRHa}$ and HRT protocol was then performed as previously described.

\section{Luteal Phase Support And Pregnancy Confirmation}

$600 \mathrm{mg}$ of vaginal micronized progesterone (Utrogestan, Besins, Belgium), 30mg oral progesterone (Dupbaston, Abbott Biologicals B.V., Netherlands) daily, and estrogens (the same dosage as endometrial preparation) were given from the day of embryo transfer. All patients were followed up and the pregnancy test with biochemical hCG was performed 14 days after embryo transfer. If serum hCG > 50 IU/L, luteal support continued. Transvaginal ultrasound was performed 5 weeks after embryo transfer to determine the number of gestational sacs and fetal viability.

\section{Definition Of Clinical Outcomes}

According to the ASRM consensus published in 2017, the definition of clinical outcomes was confirmed [7]. Clinical pregnancy was defined as an intrauterine gestational sac and fetal heart motion diagnosed by ultrasonographic visualization. Preterm birth was defined as a birth that occurs before 37 weeks of gestation. Ectopic pregnancy was defined as a gestational sac observed by ultrasound outside the uterine cavity. Miscarriage was defined as a pregnancy loss before 24 weeks. Live birth was defined as the delivery of any viable neonate beyond 24 weeks of gestation. All the patients were followed up until one year after embryo transfer.

\section{Statistical analysis}

Data are presented as mean \pm SD for continuous variables and as $n(\%)$ for categoric variables. Counts and proportions were used for the categorical variables. One-way analysis of variance was used for continuous variables, and the Pearson's $\chi^{2}$ test was used for categorical variables with Fisher's exact test when necessary. Logistic regression, presented as unadjusted odds ratio (crude odds ratio (OR)) or adjusted odds ratio (aOR) with 95\% confidence interval (Cl) with the 95\% of confidence interval (Cl), was performed. A variance inflation factor (VIF) was calculated to test for collinearity among the predictors. We adjusted for covariates that, when added to this model, changed the matched odds ratio by at least 10 percent in the multivariable regression analyses. In the adjusted model: we adjusted female age at embryo transfer, female age at oocyte retrieval, infertility duration, infertility type, cause of infertility, AFC, $\mathrm{BMI}$, endometrial thickness, duration of endometrial preparation, triple-line endometrial pattern, protocol in the fresh cycle, Gn duration, Gn dosage, no. of oocyte retrieved, no. of transferred embryos, no. of available embryos, no. of good quality embryos transferred, embryo stage and fertilization type. Data were analyzed using the statistical packages R (The R Foundation; http://www.r-project.org;version 3.4.3) and Empower (R) (www.empowerstates.com, X\&Y solutions, inc. Boston, Massachusetts). The level of significance was set at $p<0.05$. 


\section{Results}

A total of 1638 PCOS cycles of frozen-thawed embryo transfers were performed: 1115 cycles of HRT and 523 cycles of GnRHa + HRT. In the propensity score-matched cohort, 519 patients using GnRHa + HRT were matched with 519 patients using HRT. The patients' baseline characteristics were described in Table 1. The female age at embryo transfer was higher in the GnRHa + HRT group than HRT $(29.08 \pm 3.16$ vs $28.61 \pm 3.38, p=0.007)$. Gonadotropin $(G n)$ dosage ( $2135.55 \pm 979.92$ vs $2001.76 \pm 891.03, p=0.006)$ and $G$ duration $(12.62 \pm 8.31$ vs $10.89 \pm 3.12, p<0.001)$ in fresh cycles were higher in GnRHa + HRT group than HRT. Other demographic characteristic was similar between the two groups. After matching, the baseline characteristics of the patients were similar in the two groups. 
Table 1

Baseline characteristics between different endometrial preparation groups

\begin{tabular}{|c|c|c|c|c|c|c|}
\hline \multirow[b]{2}{*}{ Variable } & \multicolumn{3}{|c|}{ Matched Cohort $(n=1038)^{a}$} & \multicolumn{3}{|c|}{ Entire Cohort $(n=1638)^{b}$} \\
\hline & $\begin{array}{l}\text { HRT } \\
519)\end{array}(n=$ & $\begin{array}{l}\mathrm{GnRHa}+ \\
\mathrm{HRT}(\mathrm{n}= \\
519)\end{array}$ & $\begin{array}{l}P- \\
\text { value }^{9}\end{array}$ & $\begin{array}{l}\text { HRT }(n= \\
1115)\end{array}$ & $\begin{array}{l}\mathrm{GnRHa}+ \\
\mathrm{HRT}(\mathrm{n}= \\
523)\end{array}$ & $\begin{array}{l}P- \\
\text { value }\end{array}$ \\
\hline $\begin{array}{l}\text { Female age at oocyte } \\
\text { retrieval (years) }\end{array}$ & $\begin{array}{l}28.08 \pm \\
1.99\end{array}$ & $28.51 \pm 3.16$ & 0.284 & $\begin{array}{l}28.16 \pm \\
3.35\end{array}$ & $\begin{array}{l}28.49 \pm \\
3.17\end{array}$ & 0.059 \\
\hline $\begin{array}{l}\text { Female age at } \\
\text { embryo transfer } \\
\text { (years) }\end{array}$ & $\begin{array}{l}28.15 \pm \\
2.07\end{array}$ & $29.01 \pm 3.17$ & 0.132 & $\begin{array}{l}28.61 \pm \\
3.38\end{array}$ & $\begin{array}{l}29.08 \pm \\
3.16\end{array}$ & 0.007 \\
\hline $\begin{array}{l}\text { Infertility } \\
\text { duration(years) }\end{array}$ & $\begin{array}{l}3.53 \pm \\
1.59\end{array}$ & $3.61 \pm 2.13$ & 0.431 & $\begin{array}{l}3.59 \pm \\
2.29\end{array}$ & $3.61 \pm 2.13$ & 0.896 \\
\hline $\mathrm{BMI}\left(\mathrm{kg} / \mathrm{m}^{2}\right)$ & $\begin{array}{l}23.92 \pm \\
3.24\end{array}$ & $24.12 \pm 3.74$ & 0.182 & $\begin{array}{l}23.96 \pm \\
3.49\end{array}$ & $\begin{array}{l}24.12 \pm \\
3.74\end{array}$ & 0.396 \\
\hline AFC (n) & $\begin{array}{l}22.41 \pm \\
3.14\end{array}$ & $22.51 \pm 3.32$ & 0.804 & $\begin{array}{l}22.30 \pm \\
3.36\end{array}$ & $\begin{array}{l}22.49 \pm \\
3.35\end{array}$ & 0.285 \\
\hline $\begin{array}{l}\text { No. of oocyte } \\
\text { retrieved }(n)\end{array}$ & $\begin{array}{l}19.14 \pm \\
8.68\end{array}$ & $18.66 \pm 7.55$ & 0.083 & $\begin{array}{l}19.95 \pm \\
8.30\end{array}$ & $\begin{array}{l}17.65 \pm \\
7.54\end{array}$ & $<001$ \\
\hline $\begin{array}{l}\text { Fertilization type (n, } \\
\%)\end{array}$ & & & $0.363^{\ddagger}$ & & & $0.871^{\ddagger}$ \\
\hline IVF & $\begin{array}{l}410 \\
(79.00 \%)\end{array}$ & $\begin{array}{l}417 \\
(80.35 \%)\end{array}$ & & $\begin{array}{l}904 \\
(81.08 \%)\end{array}$ & $\begin{array}{l}421 \\
(80.50 \%)\end{array}$ & \\
\hline ICSI & $\begin{array}{l}94 \\
(18.11 \%)\end{array}$ & $81(15.61 \%)$ & & $\begin{array}{l}172 \\
(15.43 \%)\end{array}$ & $\begin{array}{l}81 \\
(15.49 \%)\end{array}$ & \\
\hline IVF + ICSI & $\begin{array}{l}15 \\
(2.89 \%)\end{array}$ & $21(4.05 \%)$ & & $\begin{array}{l}39 \\
(3.50 \%)\end{array}$ & $21(4.02 \%)$ & \\
\hline Infertility type (n, \%) & & & $0.007^{\ddagger}$ & & & $0.647^{\ddagger}$ \\
\hline Primary infertility & $\begin{array}{l}407 \\
(78.72 \%)\end{array}$ & $\begin{array}{l}370 \\
(71.43 \%)\end{array}$ & & $\begin{array}{l}783 \\
(70.35 \%)\end{array}$ & $\begin{array}{l}373 \\
(71.46 \%)\end{array}$ & \\
\hline Secondary infertility & $\begin{array}{l}110 \\
(21.28 \%)\end{array}$ & $\begin{array}{l}148 \\
(28.57 \%)\end{array}$ & & $\begin{array}{l}330 \\
(29.65 \%)\end{array}$ & $\begin{array}{l}149 \\
(28.54 \%)\end{array}$ & \\
\hline $\begin{array}{l}\text { Cause of infertility (n, } \\
\%)\end{array}$ & & & $0.363^{\ddagger}$ & & & $0.371^{\ddagger}$ \\
\hline Tubal & $\begin{array}{l}42 \\
(8.09 \%)\end{array}$ & $21(4.05 \%)$ & & $\begin{array}{l}69 \\
(6.19 \%)\end{array}$ & $22(4.21 \%)$ & \\
\hline \multicolumn{7}{|l|}{${ }^{\ddagger} \chi 2$ test, 'Student's $t$ test } \\
\hline
\end{tabular}




\begin{tabular}{|c|c|c|c|c|c|c|}
\hline \multirow[b]{2}{*}{ Ovulation disorder } & \multicolumn{3}{|c|}{ Matched Cohort $(n=1038)^{a}$} & \multicolumn{3}{|c|}{ Entire Cohort $(n=1638)^{b}$} \\
\hline & $\begin{array}{l}145 \\
(27.94 \%)\end{array}$ & $\begin{array}{l}186 \\
(35.84 \%)\end{array}$ & & $\begin{array}{l}388 \\
(34.83 \%)\end{array}$ & $\begin{array}{l}186 \\
(35.56 \%)\end{array}$ & \\
\hline Male factor & $\begin{array}{l}18 \\
(3.47 \%)\end{array}$ & 11 (2.12\%) & & $\begin{array}{l}29 \\
(2.60 \%)\end{array}$ & 11 (2.10\%) & \\
\hline Mixed factor & $\begin{array}{l}314 \\
(60.50 \%)\end{array}$ & $\begin{array}{l}301 \\
(58.00 \%)\end{array}$ & & $\begin{array}{l}628 \\
(56.37 \%)\end{array}$ & $\begin{array}{l}304 \\
(58.13 \%)\end{array}$ & \\
\hline $\begin{array}{l}\text { Protocol in the fresh } \\
\text { cycle }(n, \%)\end{array}$ & & & $0.210^{\ddagger}$ & & & $0.477^{\ddagger}$ \\
\hline Agonist & $\begin{array}{l}462 \\
(89.02 \%)\end{array}$ & $\begin{array}{l}478 \\
(92.10 \%)\end{array}$ & & $\begin{array}{l}1007 \\
(90.31 \%)\end{array}$ & $\begin{array}{l}482 \\
(92.16 \%)\end{array}$ & \\
\hline Antagonist & $\begin{array}{l}55 \\
(10.60 \%)\end{array}$ & $40(7.71 \%)$ & & $\begin{array}{l}105 \\
(9.42 \%)\end{array}$ & $40(7.65 \%)$ & \\
\hline Other & $2(0.39 \%)$ & $1(0.19 \%)$ & & $\begin{array}{l}3 \\
(0.27 \%)\end{array}$ & $1(0.19 \%)$ & \\
\hline Gn dosage (IU) & $\begin{array}{l}2006.81 \\
\pm 734.87\end{array}$ & $\begin{array}{l}2142.23 \pm \\
978.10\end{array}$ & 0.082 & $\begin{array}{l}2001.76 \\
\pm 891.03\end{array}$ & $\begin{array}{l}2135.55 \pm \\
979.92\end{array}$ & 0.006 \\
\hline Gn duration (days) & $\begin{array}{l}10.59 \pm \\
3.00\end{array}$ & $10.66 \pm 8.32$ & 0.371 & $\begin{array}{l}10.89 \pm \\
3.12\end{array}$ & $\begin{array}{l}12.62 \pm \\
8.31\end{array}$ & $<.001$ \\
\hline \multicolumn{7}{|c|}{${ }^{\ddagger} \chi 2$ test, 'Student's $t$ test } \\
\hline
\end{tabular}

Outcomes of two groups for endometrial preparation were shown in Table 2. The endometrial thickness on the day of $\mathrm{P}$ administration was thicker in the GnRHa + HRT group (10.18 \pm 1.55 vs $9.76 \pm 1.54, p<$ 0.001). Patients in GnRHa + HRT group transferred more cleavage stage embryos than HRT (33.3\% vs $25.3 \%, p<0.001)$. No. of the available embryos in the GnRHa + HRT group were lesser than HRT. The preterm birth rate was higher in $\mathrm{GnRHa}+\mathrm{HRT}$ than HRT $(20.08 \%$ vs $14.08 \%, \mathrm{p}=0.002)$. Clinical pregnancy rate, miscarriage rate, ectopic pregnancy rate, the live birth rate was not statistically different between the two groups before and after PSM. 
Table 2

Outcomes of two groups for endometrial preparation

\begin{tabular}{|c|c|c|c|c|c|c|}
\hline \multirow[b]{2}{*}{ Variable } & \multicolumn{3}{|c|}{ Matched Cohort $(n=1038)^{a}$} & \multicolumn{3}{|c|}{ Entire Cohort $(n=1638)^{b}$} \\
\hline & $\begin{array}{l}\text { HRT }(n= \\
519)\end{array}$ & $\begin{array}{l}\text { GnRHa + } \\
\operatorname{HRT}(n= \\
519)\end{array}$ & $\begin{array}{l}P- \\
\text { value }\end{array}$ & $\begin{array}{l}\operatorname{HRT}(n= \\
1115)\end{array}$ & $\begin{array}{l}\text { GnRHa } \\
+\mathrm{HRT}(\mathrm{n} \\
=523)\end{array}$ & $\begin{array}{l}P \text { - } \\
\text { value }\end{array}$ \\
\hline $\begin{array}{l}\text { Duration of endometrial } \\
\text { preparation (days) }\end{array}$ & $\begin{array}{l}11.14 \pm \\
3.59\end{array}$ & $\begin{array}{l}11.61 \pm \\
3.59\end{array}$ & 0.032 & $\begin{array}{l}11.35 \pm \\
3.77\end{array}$ & $\begin{array}{l}11.61 \pm \\
3.58\end{array}$ & 0.470 \\
\hline $\begin{array}{l}\text { Endometrial thickness on } \\
\text { the day of } P \\
\text { administration }(\mathrm{mm})\end{array}$ & $\begin{array}{l}9.80 \pm \\
1.50\end{array}$ & $\begin{array}{l}10.18 \pm \\
1.55\end{array}$ & $<.001$ & $\begin{array}{l}9.76 \pm \\
1.54\end{array}$ & $\begin{array}{l}10.18 \pm \\
1.55\end{array}$ & $\dot{0} 001$ \\
\hline $\begin{array}{l}\text { Triple-line endometrial } \\
\text { pattern (\%) }\end{array}$ & & & $0.189^{\ddagger}$ & & & $0.008^{\ddagger}$ \\
\hline A & $\begin{array}{l}60 \\
(11.56 \%)\end{array}$ & $\begin{array}{l}75 \\
(14.45 \%)\end{array}$ & & $\begin{array}{l}103 \\
(9.24 \%)\end{array}$ & $\begin{array}{l}75 \\
(14.34 \%)\end{array}$ & \\
\hline B & $\begin{array}{l}432 \\
(83.24 \%)\end{array}$ & $\begin{array}{l}409 \\
(78.81 \%)\end{array}$ & & $\begin{array}{l}931 \\
(83.50 \%)\end{array}$ & $\begin{array}{l}413 \\
(78.97 \%)\end{array}$ & \\
\hline C & $\begin{array}{l}27 \\
(5.20 \%)\end{array}$ & $\begin{array}{l}35 \\
(6.74 \%)\end{array}$ & & $\begin{array}{l}81 \\
(7.26 \%)\end{array}$ & $\begin{array}{l}35 \\
(6.69 \%)\end{array}$ & \\
\hline Embryo stage $(n, \%)$ & & & $0.189^{\ddagger}$ & & & $\begin{array}{l}< \\
0.001^{\ddagger}\end{array}$ \\
\hline Cleavage stage & $\begin{array}{l}140 \\
(26.97 \%)\end{array}$ & $\begin{array}{l}173 \\
(33.33 \%)\end{array}$ & & $\begin{array}{l}282 \\
(25.29 \%)\end{array}$ & $\begin{array}{l}174 \\
(33.27 \%)\end{array}$ & \\
\hline Blastocyst stage & $\begin{array}{l}379 \\
(73.03 \%)\end{array}$ & $\begin{array}{l}346 \\
(66.67 \%)\end{array}$ & & $\begin{array}{l}833 \\
(74.71 \%)\end{array}$ & $\begin{array}{l}349 \\
(66.73 \%)\end{array}$ & \\
\hline $\begin{array}{l}\text { No. of transferred } \\
\text { embryos }(n, \%)\end{array}$ & $\begin{array}{l}10.10 \pm \\
5.18\end{array}$ & $\begin{array}{l}8.69 \pm \\
4.69\end{array}$ & 0.725 & $\begin{array}{l}1.74 \pm \\
0.46\end{array}$ & $\begin{array}{l}1.76 \pm \\
0.45\end{array}$ & 0.428 \\
\hline $\begin{array}{l}\text { No. of the available } \\
\text { embryos }(n, \%)\end{array}$ & $\begin{array}{l}10.10 \pm \\
5.18\end{array}$ & $\begin{array}{l}8.69 \pm \\
4.69\end{array}$ & $<.001$ & $\begin{array}{l}10.08 \pm \\
5.26\end{array}$ & $\begin{array}{l}8.69 \pm \\
4.70\end{array}$ & $<.001$ \\
\hline $\begin{array}{l}\text { No. of good quality } \\
\text { embryos transferred (n, \%) }\end{array}$ & $\begin{array}{l}1.16 \pm \\
0.77\end{array}$ & $\begin{array}{l}1.06 \pm \\
0.78\end{array}$ & 0.049 & $\begin{array}{l}1.15 \pm \\
0.75\end{array}$ & $\begin{array}{l}1.06 \pm \\
0.78\end{array}$ & 0.025 \\
\hline $\begin{array}{l}\text { Clinical pregnancy rate }(\mathrm{n}, \\
\%)\end{array}$ & $\begin{array}{l}401 \\
(77.26 \%)\end{array}$ & $\begin{array}{l}391 \\
(75.34 \%)\end{array}$ & $0.465^{\ddagger}$ & $\begin{array}{l}811 \\
(72.74 \%)\end{array}$ & $\begin{array}{l}394 \\
(75.33 \%)\end{array}$ & $0.266^{\ddagger}$ \\
\hline Preterm birth rate $(\mathrm{n}, \%)$ & $\begin{array}{l}79 \\
(15.22 \%)\end{array}$ & $\begin{array}{l}104 \\
(20.04 \%)\end{array}$ & $0.042^{\ddagger}$ & $\begin{array}{l}157 \\
(14.08 \%)\end{array}$ & $\begin{array}{l}105 \\
(20.08 \%)\end{array}$ & $0.002^{\ddagger}$ \\
\hline
\end{tabular}

\section{${ }^{\ddagger} \chi 2$ test, 'Student's $t$ test}

a Propensity score-matched cohort, ${ }^{b}$ before propensity score matching. 


\begin{tabular}{|c|c|c|c|c|c|c|}
\hline \multirow[b]{2}{*}{ Miscarriage rate $(\mathrm{n}, \%)$} & \multicolumn{3}{|c|}{ Matched Cohort $(n=1038)^{a}$} & \multicolumn{3}{|c|}{ Entire Cohort $(n=1638)^{b}$} \\
\hline & $\begin{array}{l}71 \\
(13.68 \%)\end{array}$ & $\begin{array}{l}73 \\
(14.07 \%)\end{array}$ & $0.857^{\ddagger}$ & $\begin{array}{l}159 \\
(14.26 \%)\end{array}$ & $\begin{array}{l}73 \\
(13.96 \%)\end{array}$ & $0.870^{\ddagger}$ \\
\hline $\begin{array}{l}\text { Ectopic pregnancy rate (n, } \\
\%)\end{array}$ & $\begin{array}{l}3 \\
(0.58 \%)\end{array}$ & $0(0.00 \%)$ & $0.654^{\ddagger}$ & $\begin{array}{l}4 \\
(0.36 \%)\end{array}$ & $\begin{array}{l}2 \\
(0.38 \%)\end{array}$ & $0.941^{\ddagger}$ \\
\hline Live birth rate $(\mathrm{n}, \%)$ & $\begin{array}{l}314 \\
(60.50 \%)\end{array}$ & $\begin{array}{l}313 \\
(60.31 \%)\end{array}$ & $0.949^{\ddagger}$ & $\begin{array}{l}619 \\
(55.52 \%)\end{array}$ & $\begin{array}{l}316 \\
(60.42 \%)\end{array}$ & $0.062^{\ddagger}$ \\
\hline Birth weight $(\mathrm{kg})$ & $\begin{array}{l}3.07 \pm \\
0.68\end{array}$ & $\begin{array}{l}3.07 \pm \\
0.68\end{array}$ & 0.830 & $\begin{array}{l}3.07 \pm \\
0.72\end{array}$ & $\begin{array}{l}3.07 \pm \\
0.68\end{array}$ & 0.914 \\
\hline Fetal sex $(n, \%)$ & & & $0.403^{\ddagger}$ & & & $0.783^{\ddagger}$ \\
\hline Female & $\begin{array}{l}161 \\
(51.27 \%)\end{array}$ & $\begin{array}{l}151 \\
(47.94 \%)\end{array}$ & & $\begin{array}{l}290 \\
(46.85 \%)\end{array}$ & $\begin{array}{l}152 \\
(47.80 \%)\end{array}$ & \\
\hline Male & $\begin{array}{l}153 \\
(48.73 \%)\end{array}$ & $\begin{array}{l}164 \\
(52.06 \%)\end{array}$ & & $\begin{array}{l}329 \\
(53.15 \%)\end{array}$ & $\begin{array}{l}166 \\
(52.20 \%)\end{array}$ & \\
\hline \multicolumn{7}{|l|}{${ }^{\ddagger} \chi 2$ test, 'Student's $t$ test } \\
\hline
\end{tabular}

Univariate analysis was performed to evaluate each variable's effect on the live birth rate (Table 3). BMI and AFC were negatively associated with live birth, while endometrial thickness on the day of $P$ administration, no. of the available embryos, no. of transferred embryos, no. of good quality embryos transferred, and blastocyst stage embryo transfer was positively associated with live birth. 
Table 3

Univariate analysis for live birth rate in the entire cohort

\begin{tabular}{|c|c|c|c|}
\hline Covariate & Statistics & OR $(95 \% \mathrm{Cl})$ & $\begin{array}{l}P \\
\text { value }\end{array}$ \\
\hline Female age at oocyte retrieval (years) & $28.26 \pm 3.30$ & $0.997(0.97,1.03)$ & 0.859 \\
\hline Female age at embryo transfer (years) & $28.76 \pm 3.32$ & $0.99(0.96,1.02)$ & 0.446 \\
\hline $\mathrm{BMI}\left(\mathrm{kg} / \mathrm{m}^{2}\right)$ & $24.01 \pm 3.57$ & $0.93(0.91,0.96)$ & $<.001$ \\
\hline Infertility duration (years) & $3.60 \pm 2.24$ & $0.97(0.93,1.01)$ & 0.154 \\
\hline \multicolumn{4}{|l|}{ Infertility type (\%) } \\
\hline primary infertility & 70.70 & Reference & \\
\hline secondary infertility & 29.30 & $0.95(0.76,1.17)$ & $0.611^{\ddagger}$ \\
\hline $\operatorname{AFC}(n)$ & $22.36 \pm 3.36$ & $0.97(0.94,0.99)$ & 0.020 \\
\hline $\begin{array}{l}\text { Endometrial thickness on the day of } P \\
\text { administration }(\mathrm{mm})\end{array}$ & $9.90 \pm 1.55$ & $1.14(1.07,1.22)$ & $<.001$ \\
\hline \multicolumn{4}{|l|}{ Fertilization type $(\mathrm{n}, \%)$} \\
\hline IVF & $\begin{array}{l}1325 \\
(80.89 \%)\end{array}$ & Reference & \\
\hline ICSI & 253 (15.45\%) & $0.77(0.59,1.01)$ & $0.057^{\ddagger}$ \\
\hline IVF + ICSI & $60(3.66 \%)$ & $0.82(0.49,1.38)$ & $0.450^{\ddagger}$ \\
\hline \multicolumn{4}{|l|}{ Protocol in the fresh cycle $(n, \%)$} \\
\hline Agonist & $\begin{array}{l}1489 \\
(90.90 \%)\end{array}$ & Reference & \\
\hline Antagonist & 145 (8.85\%) & $0.72(0.51,1.01)$ & $0.057^{\ddagger}$ \\
\hline Other & $4(0.24 \%)$ & $0.24(0.03,2.34)$ & $0.220^{\ddagger}$ \\
\hline Gn duration (days) & $11.44 \pm 5.41$ & $1.002(0.98,1.02)$ & 0.804 \\
\hline Gn dosage (IU) & $\begin{array}{l}2044.48 \pm \\
922.16\end{array}$ & $\begin{array}{l}0.9999(0.9998 \\
1.0000)\end{array}$ & 0.147 \\
\hline No. of oocyte retrieved $(n)$ & $19.22 \pm 8.14$ & $1.01(1.00,1.02)$ & 0.151 \\
\hline No. of the available embryos (n) & $9.64 \pm 5.12$ & $1.03(1.01,1.05)$ & 0.001 \\
\hline \multicolumn{4}{|l|}{ No. of transferred embryos (n, \%) } \\
\hline${ }^{\ddagger} \chi 2$ test, 'Student's $t$ test & & & \\
\hline
\end{tabular}




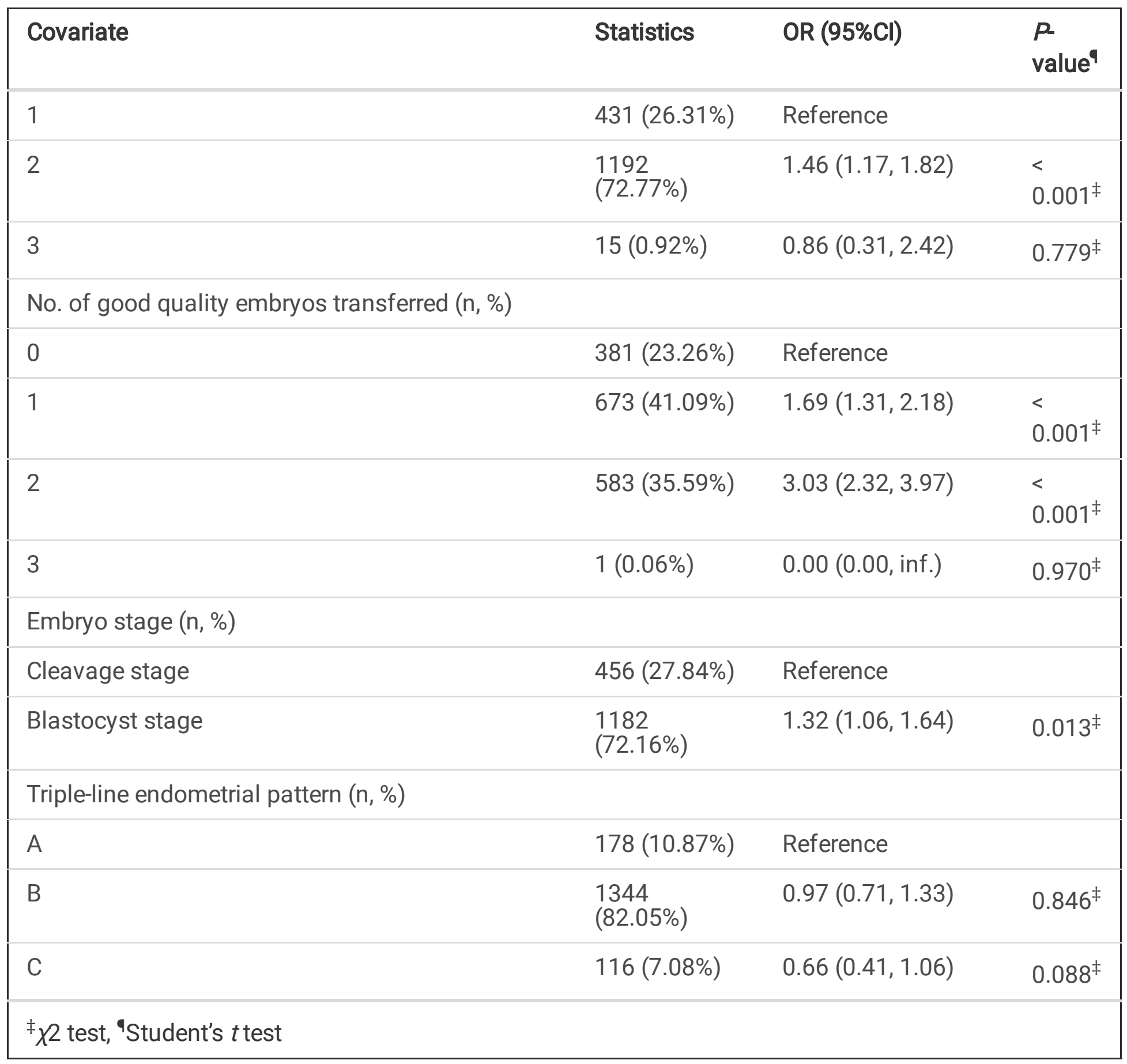

Table 4 showed effect size of endometrial preparation on live birth rate in prespecified and exploratory subgroups in each subgroup. No significant interactions were found in any of the subgroups. 
Table 4

Effect size of endometrial preparation on live birth rate in prespecified and exploratory subgroups in each subgroup

\begin{tabular}{|c|c|c|c|c|}
\hline Characteristic & $\begin{array}{l}\text { No. of } \\
\text { participants }\end{array}$ & $\begin{array}{l}\mathrm{OR}(95 \% \mathrm{Cl}) \\
\mathrm{a}\end{array}$ & $\begin{array}{l}P \\
\text { value }\end{array}$ & $\begin{array}{l}P \text { for } \\
\text { interaction }\end{array}$ \\
\hline $\begin{array}{l}\text { Endometrial thickness on the day of } P \\
\text { administration }(\mathrm{mm})\end{array}$ & & & & 0.952 \\
\hline Tertile $1(<9.00)$ & 531 & $\begin{array}{l}1.11(0.74 \\
1.67)\end{array}$ & 0.609 & \\
\hline Tertile 2 (9.00-10.30) & 537 & $\begin{array}{l}1.22(0.85 \\
1.75)\end{array}$ & 0.292 & \\
\hline Tertile 3 (> 10.30) & 570 & $\begin{array}{l}1.16(0.82 \\
1.64)\end{array}$ & 0.404 & \\
\hline \multicolumn{5}{|l|}{ Triple-line endometrial pattern } \\
\hline A & 178 & $\begin{array}{l}1.02(0.56 \\
1.86)\end{array}$ & 0.956 & 0.169 \\
\hline B & 1344 & $\begin{array}{l}1.19(0.94 \\
1.50)\end{array}$ & 0.156 & \\
\hline C & 116 & $\begin{array}{l}2.34(1.04 \\
5.29)\end{array}$ & 0.041 & \\
\hline \multicolumn{5}{|l|}{ No. of transferred embryos } \\
\hline 1 & 431 & $\begin{array}{l}0.94(0.62 \\
1.41)\end{array}$ & 0.760 & 0.227 \\
\hline 2 & 1192 & $\begin{array}{l}1.34(1.05 \\
1.73)\end{array}$ & 0.021 & \\
\hline 3 & 15 & $\begin{array}{l}1.25(0.16 \\
9.92)\end{array}$ & 0.833 & \\
\hline \multicolumn{5}{|l|}{ Female age at embryo transfer } \\
\hline Tertile $1(<27)$ & 428 & $\begin{array}{l}1.15(0.74 \\
1.79)\end{array}$ & 0.529 & 0.311 \\
\hline Tertile 2 (27-29) & 591 & $\begin{array}{l}1.00(0.71 \\
1.42)\end{array}$ & 0.978 & \\
\hline Tertile 3 (> 29) & 619 & $\begin{array}{l}1.51(1.08 \\
2.13)\end{array}$ & 0.017 & \\
\hline
\end{tabular}

\section{${ }^{\ddagger} \chi 2$ test, ${ }^{9}$ Student's $t$ test}

a Adjusted for AFC, BMI, infertility duration, infertility type and cause of infertility except the subgroup variable. 


\begin{tabular}{|c|c|c|c|c|}
\hline Characteristic & $\begin{array}{l}\text { No. of } \\
\text { participants }\end{array}$ & $\underset{a}{\mathrm{OR}}(95 \% \mathrm{Cl})$ & $\begin{array}{l}P \\
\text { value }^{\ddagger}\end{array}$ & $\begin{array}{l}P \text { for } \\
\text { interaction }\end{array}$ \\
\hline \multicolumn{5}{|c|}{ Fertilization type } \\
\hline IVF & 1325 & $\begin{array}{l}1.17(0.92 \\
1.48)\end{array}$ & 0.200 & 0.257 \\
\hline ICSI & 253 & $\begin{array}{l}1.25(0.74 \\
2.12)\end{array}$ & 0.410 & \\
\hline IVF + ICSI & 60 & $\begin{array}{l}3.24(1.04 \\
10.10)\end{array}$ & 0.043 & \\
\hline \multicolumn{5}{|c|}{ Protocol in the fresh cycle } \\
\hline Agonist & 1489 & $\begin{array}{l}1.22(0.98 \\
1.53)\end{array}$ & 0.074 & 0.849 \\
\hline Antagonist & 145 & $\begin{array}{l}1.02(0.49, \\
2.11)\end{array}$ & 0.959 & \\
\hline Other & 4 & & & \\
\hline \multicolumn{5}{|c|}{${ }^{\ddagger} \chi 2$ test, 'Student's $t$ test } \\
\hline $\begin{array}{l}\text { a Adjusted for } \\
\text { variable. }\end{array}$ & ility type anc & Ise of infert & except & e subgroup \\
\hline
\end{tabular}

The multiple logistic regression model showed no difference between endometrial preparation and live birth while adjusting for potential confounders presented in Table 5. After adjusting for covariates, no difference was found in live birth between $\mathrm{GnRHa}+\mathrm{HRT}$ and HRT before (OR 1.56, 95\% $\mathrm{Cl}, 1.001-2.41$, p $=0.048)$ and after PSM (OR 0.88, 95\%Cl, $0.38-2.02, \mathrm{p}=0.763)$. 
Table 5

Relationship between endometrial preparation and live birth in different models

\begin{tabular}{|c|c|c|c|c|c|c|}
\hline \multirow[t]{2}{*}{ Outcome } & \multicolumn{2}{|c|}{ Crude Model ${ }^{a}$} & \multicolumn{2}{|c|}{ Adjusted Model ${ }^{b}$} & \multicolumn{2}{|c|}{ PSM-Model c } \\
\hline & OR $(95 \% \mathrm{Cl})$ & $\begin{array}{l}P \\
\text { value }^{\ddagger}\end{array}$ & OR $(95 \% \mathrm{Cl})$ & $\begin{array}{l}P \\
\text { value }^{\ddagger}\end{array}$ & OR $(95 \% \mathrm{Cl})$ & $\begin{array}{l}P \\
\text { value }\end{array}$ \\
\hline \multicolumn{7}{|l|}{$\begin{array}{l}\text { Endometrial } \\
\text { preparation }\end{array}$} \\
\hline HRT & Reference & & Reference & & Reference & \\
\hline $\mathrm{GnRHa}+\mathrm{HRT}$ & $\begin{array}{l}1.22(0.99 \\
1.51)\end{array}$ & 0.062 & $\begin{array}{l}1.56(1.001 \\
2.41)\end{array}$ & 0.048 & $\begin{array}{l}0.88(0.38 \\
2.02)\end{array}$ & 0.763 \\
\hline \multicolumn{7}{|c|}{${ }^{\ddagger} \chi 2$ test, 'Student's $t$ test } \\
\hline \multicolumn{7}{|c|}{ a We did not adjust other covariates in entire cohort. } \\
\hline \multicolumn{7}{|c|}{$\begin{array}{l}\text { b Adjusted for female age at embryo transfer, female age at oocyte retrieval, infertility duration, } \\
\text { infertility type, cause of infertility, AFC, BMI, endometrial thickness, duration of endometrial } \\
\text { preparation, triple-line endometrial pattern, protocol in the fresh cycle, Gn duration, Gn dosage, no. of } \\
\text { oocyte retrieved, no. of transferred embryos, no. of the available embryos, no. of good quality } \\
\text { embryos transferred, embryo stage and fertilization type in entire cohort. }\end{array}$} \\
\hline${ }^{\mathrm{C}}$ Matched col & & & & & & \\
\hline
\end{tabular}

\section{Discussion}

The most advantageous protocol for FET has been the subject of several retrospective studies. To the best of our knowledge, we present the largest analysis to date that compares pretreatment with and without GnRHa with hormone protocol for PCOS patients. Our data suggest that endometrial preparation with GnRHa pretreatment does not increase the live birth rate of PCOS patients undergoing FET.

Despite the different experiences and doctors' preferences with endometrial preparation, there is a lack of evidence to support the superiority of one method over the other. In the past, the most popular protocol of endometrial preparation was HRT, to avoid spontaneous ovulation. A transfer in the HRT cycle is more applicable in women who are not menstruating regularly such as those with ovulation disorder or PCOS and in cases where a better control or a flexible transfer is indicated [8]. HRT cycles can be used with or without a GnRHa for pituitary suppression. There are a few numbers of studies comparing HRT and $\mathrm{GnRHa}+\mathrm{HRT}$ cycles, however, the results have been conflicting. A prospective randomized study indicated HRT resulted in a similar success rate and lower cost than GnRHa + HRT [8]. However, the sample size was small ( $n=106$ cycles) and did not adjust the confounding covariate. Studies have shown long-term GnRHa before IVF treatment in infertile women with endometriosis increases pregnancy rate $[9,10]$. One retrospective study involving 339 cycles of FET patients with adenomyosis and found 
long-term GnRHa pretreatment significantly improved pregnancy outcomes [11]. However, the beneficial effect of GnRHa in PCOS patients before FET is not known.

Infertile women with PCOS are at a greater risk of early pregnancy loss, aberrant uterine receptivity, and high body mass index [12-14]. When the pituitary is suppressed by the administration of $\mathrm{GnRHa}$, initial follicular activity is inhibited. HRT cycles in the absence of GnRHa suppression can result in a rise in LH, ultimately negatively altering the receptive window of implantation [15]. It can be speculated that $\mathrm{GnRHa}$ pretreatment may produce a window of time with improved implantation and increased endometrial receptivity [9, 16-17]. An additional advantage of $\mathrm{GnRHa}+\mathrm{HRT}$ is the convenience of better control over the cycle and transfer date [18]. One retrospective study showed that GnRHa pretreatment significantly increased the ongoing pregnancy rate in PCOS women [19]. Another possible mechanism underlying the benefits of GnRHa pretreatment for PCOS is suppression of LH level, $E_{2}$ level, hyperandrogenic level, and GnRH-HCG axis function with inhibition of endometrial inflammation and enhanced expression of endometrial adhesion molecules [20]. GnRHa pretreatment in PCOS patients could lead to an androgen deprivation status, in addition, subsequent HRT cycles for endometrial preparation can synchronize the development of the embryo with the endometrium. However, GnRHa pretreatment has some major disadvantages: the duration of treatment is prolonged, the women may suffer from menopausal symptoms resulting from a hypo-estrogenic state caused by the agonist, and an agonist may lead to ovarian cyst formation.

\section{Strengths And Limitations}

The main strengths of our study include the population size, the ability to perform sensitivity analysis with an adequate number of patients, and adjusting more confounding covariates. The weaknesses of our study include its retrospective design and the inherent biases therein. Although we used logistic regression to maximize confounders' effect on the outcome, it is impossible to control all the confounders.

\section{Conclusions}

Our results suggest that GnRHa + HRT protocols have a comparable chance of live birth to the HRT cycle. Well-designed, prospective clinical trials are needed to further assess the beneficial effect of $\mathrm{GnRHa}$ pretreatment of FET on PCOS patients. These studies will aid in patient counseling and in personalizing frozen-thawed embryo transfer.

\section{Declarations}

\section{Acknowledgements}

The authors thank the staff from Northwest Women's and Children's Hospital for their assistance with the data collection. 


\section{Authors' contributions}

XL designed the study. JS conducted data collection, analysis and writing the manuscript. HB conceived and designed the study.

\section{Funding}

This work was supported by National Natural Science Foundation of China (No. 81771657 http://www.nsfc.gov.cn/).

\section{Availability of data and material}

The datasets used and/or analysed during the current study are available from the corresponding author on reasonable request.

\section{Ethics approval and consent to participate}

The study was granted ethical approval by the Ethics Committee of Northwest Women's and Children's Hospital (number 2019013). All participants provided written informed consent ahead of participation.

\section{Consent for publication}

Not applicable.

\section{Competing interests}

None.

\section{References}

1. Cutler DA, Shaw AK, Pride SM, et al. A randomized controlled trial comparing lifestyle intervention to letrozole for ovulation in women with polycystic ovary syndrome: a study protocol. Trials. 2018;19:632.

2. Vuong LN, Dang VQ, Ho TM, et al. IVF Transfer of Fresh or Frozen Embryos in Women without Polycystic Ovaries. N Engl J Med. 2018;378:137-47.

3. Shi Y, Sun Y, Hao C, et al. Transfer of Fresh versus Frozen Embryos in Ovulatory Women. N Engl J Med. 2018;378:126-36.

4. Pereira N, Petrini AC, Hancock KL, et al. Fresh or Frozen Embryo Transfer in In Vitro Fertilization: An Update. Clin Obstet Gynecol. 2019;62:293-9.

5. Cutting R. Single embryo transfer for all. Best Pract Res Clin Obstet Gynaecol. 2018;53:30-7.

6. Van Voorhis B, Mejia RB. Single-embryo transfer point-it is the way forward. Fertil Steril. 2017;108:757. 
7. Zegers-Hochschild F, Adamson GD, Dyer S, et al. The International Glossary on Infertility and Fertility Care. 2017; Hum Reprod. 2017, 32: 1786 - 801.

8. Simon A, Hurwitz A, Zentner BS, et al. Transfer of frozen-thawed embryos in artificially prepared cycles with and without prior gonadotrophin-releasing hormone agonist suppression: a prospective randomized study. Hum Reprod. 1998;13:2712-7.

9. Dueholm M. Uterine adenomyosis and infertility, review of reproductive outcome after in vitro fertilization and surgery. Acta Obstet Gynecol Scand. 2017;96:715-26.

10. Park $\mathrm{CW}$, Choi $\mathrm{MH}$, Yang KM, et al. Pregnancy rate in women with adenomyosis undergoing fresh or frozen embryo transfer cycles following gonadotropin-releasing hormone agonist treatment. Clin Exp Reprod Med. 2016;43:169-73.

11. Niu Z, Chen Q, Sun Y, et al. Long-term pituitary downregulation before frozen embryo transfer could improve pregnancy outcomes in women with adenomyosis. Gynecol Endocrinol. 2013;29:1026-30.

12. Yang $X$, Quan $X$, Lan $Y$, et al. Serum chemerin level in women with PCOS and its relation with the risk of spontaneous abortion. Gynecol Endocrinol. 2018;34:864-7.

13. Sheng Y, Lu G, Liu J, et al. Effect of body mass index on the outcomes of controlled ovarian hyperstimulation in Chinese women with polycystic ovary syndrome: a multicenter, prospective, observational study. J Assist Reprod Genet. 2017;34:61-70.

14. Abu Hashim H. Twenty years of ovulation induction with metformin for PCOS; what is the best available evidence? Reprod BiomedOnline. 2016;32:44-53.

15. El-Toukhy T, Taylor A, Khalaf $\mathrm{Y}$, et al. Pituitary suppression in ultrasound-monitored frozen embryo replacement cycles. A randomised study. Hum Reprod. 2004;19:874-9.

16. Harper MJ. The implantation window. Baillieres Clin Obstet Gynaecol. 1992;6:351-71.

17. Tabibzadeh S. Molecular control of the implantation window. Hum Reprod Update. 1998;4:465-71.

18. Hill MJ, Miller KA, Frattarelli JL. A GnRH agonist and exogenous hormone stimulation protocol has a higher live-birth rate than a natural endogenous hormone protocol for frozen-thawed blastocyststage embryo transfer cycles: an analysis of 1391 cycles. Fertil Steril. 2010;93:416-22.

19. Tsai HW, Wang PH, Lin LT, et al. Using gonadotropin-releasing hormone agonist before frozen embryo transfer may improve ongoing pregnancy rates in hyperandrogenic polycystic ovary syndrome women. Gynecol Endocrinol. 2017;33:686-9.

20. Luo L, Chen M, Wen Y. L, et al. Pregnancy Outcome and Cost-Effectiveness Comparisons of Artificial Cycle-Prepared Frozen Embryo Transfer with or without Gnrh Agonist Pretreatment for Polycystic Ovary Syndrome: A Randomised Controlled Trial. BJOG. 2020;8:1471.

\section{Figures}




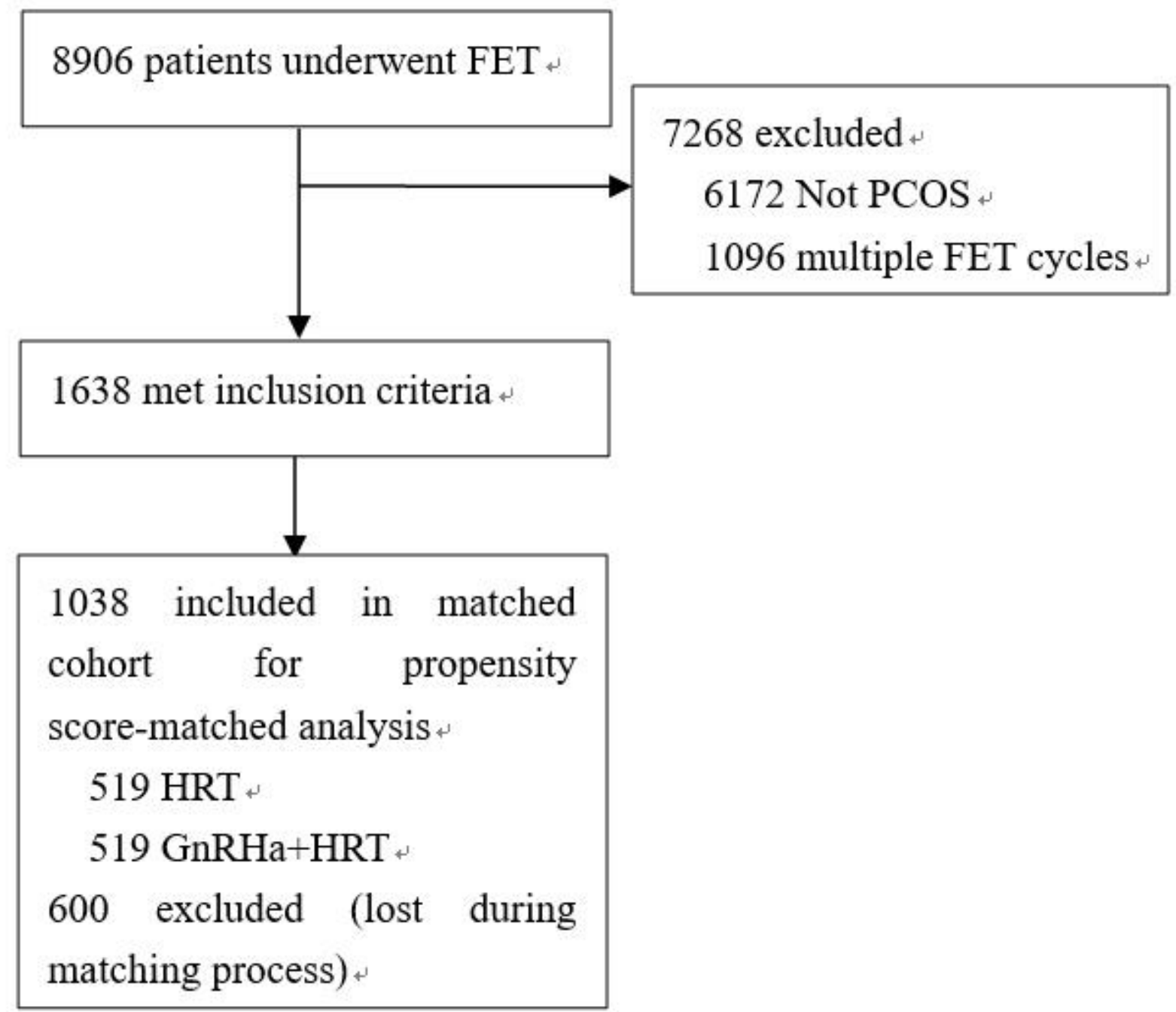

Figure 1

Selection of study population 